\title{
IMAGE COMPRESSION USING EMBEDDED ZEROTREE WAVELET
}

\author{
A.M.Raid ${ }^{1}$, W.M.Khedr ${ }^{2}$, M. A. El-dosuky ${ }^{1}$ and Wesam Ahmed ${ }^{1}$ \\ ${ }^{1}$ Mansoura University, Faculty of Computer Science and Information System \\ ${ }^{2}$ Zagazig University, Faculty of Science
}

\begin{abstract}
Compressing an image is significantly different than compressing raw binary data. compressing images is used by this different compression algorithm. Wavelet transforms used in Image compression methods to provide high compression rates while maintaining good image quality. Discrete Wavelet Transform (DWT) is one of the most common methods used in signal and image compression. It is very powerful compared to other transform because its ability to represent any type of signals both in time and frequency domain simultaneously. In this paper, we will moot the use of Wavelet Based Image compression algorithmEmbedded Zerotree Wavelet (EZW). We will obtain a bit stream with increasing accuracy from ezw algorithm because of basing on progressive encoding to compress an image into. All the numerical results were done by using matlab coding and the numerical analysis of this algorithm is carried out by sizing Peak Signal to Noise Ratio (PSNR) and Compression Ratio (CR) for standard Lena Image .Experimental results beam that the method is fast, robust and efficient enough to implement it in still and complex images with significant image compression.
\end{abstract}

\section{KEYWORDS}

Discrete wavelet Transform, embedded zerotree wavelet, Image Compression

\section{INTRODUCTION}

Multimedia data needs considerable storage capacity and transmission bandwidth. There are many forms of the data are the graphics, audio, video and image. [1]The main objective of image compression is to reduce redundancy in image data in order to store or transmit only a minimal number of samples from which a good approximation of the original image can be reconstructed in accordance with human visual perception. In general, a text file can be compressed without any errors up to original. This is called lossless compression. But after that extent errors are unavoidable. A small loss in image compression is never noticeable. In lossy compression loss and missed information is bearable. There are many types of lossless image compression include variable-length encoding, Adaptive dictionary algorithms such as LZW, bit-plane coding, lossless predictive coding, etc. Lossy compression has many classes include lossy predictive coding and transform coding. Transform coding, which applies a Fourier-related transform like DCT and Wavelet Transform like DWT are the most commonly used class. [2] Wavelet-based coding supplies fundamental improvements in image quality at higher compression ratios. Over the past few years, several competitive wavelet based Image Compression algorithms with an embedded bit stream have been developed, such as Shapiro's Embedded Zero tree Wavelet compression (EZW) algorithm, Said and Pearlman's Set Partitioning In Hierarchical Trees (SPIHT) algorithm , Truman's Embedded Block Coding with Optimized Truncation (EBCOT) algorithm Wavelet

DOI : 10.5121/sipij.2014.5603 
Signal \& Image Processing : An International Journal (SIPIJ) Vol.5, No.6, December 2014

Difference Reduction (WDR) and Adaptively Scanned Wavelet Difference Reduction (ASWDR) etc.[3][4] no many of research work has been published in literature on image compression techniques using zerotree wavelet transform so this paper will contribute to the implementation of the of lossy image compression technique (EZW algorithm).

The rest of the paper is organized as follows: section 2 explains image compression, section 3 explains (discrete wavelet transform (DWT)), section4 explains Embedded Zero tree Wavelet compression (EZW) algorithm, section5 includes simulated results and section 5 gives the conclusion and future work.

\section{IMAGE COMPRESSION}

Image Compression classifies the problem of reducing the amount of data required to represent the digital image. The time is reduced by the reduction in file size required for image to be sent over the internet or downloaded from web pages. Compression is achieved by the exit of one or more of three basic data redundancies:

(1) Spatial Redundancy .

(2) Spectral redundancy .

(3) Psycho-visual redundancy .

The spatial and spectral redundancies are present because certain spatial and spectral patterns between the pixels and the color components are common to each other, whereas the psychovisual redundancy originates from the fact that the human eye is insensitive to certain spatial frequencies. [5]

\subsection{Performance Criteria in Image Compression}

The performance is rated by the following two Essential criteria: the obtained compression ratio $\mathrm{CR}$ and the quality of the reconstructed image PSNR

(a) Compression ratio

$$
\mathrm{CR}=\mathrm{n} 1 / \mathrm{n} 2
$$

(b) Mean Square Error (MSE)

$$
M S E=\frac{1}{H W} \sum_{i=1}^{H} \sum_{j=1}^{W}[X(i, j)-Y(i, j)]^{2}
$$

(b) PSNR

$$
P S N R=10 \log _{10} \frac{255^{2}}{M S E}(d B)
$$


Signal \& Image Processing : An International Journal (SIPIJ) Vol.5, No.6, December 2014

\section{DISCRETE WAVELET TRANSFORMATION}

The Wavelet Transform used to represent a time-frequency of the signal. It was developed to cope the short coming of the Short Time Fourier Transform (STFT). The Wavelet Transform is multi-resolution technique because the different frequencies are analyzed with different resolutions.

Wavelet is one of lossy compression techniques have three procedures in general:

(1) Transform: we get a transformed data into wavelet domain. This step is invertible.

(2) Quantization: the wavelet coefficients are quantized to a limited alphabet. This step is not Invertible.

(3) Entropy Coding: the resulting symbols after quantization are further compressed to minimize the bit rate. This step is also invertible.

This simple diagram is a Wavelet Transform :

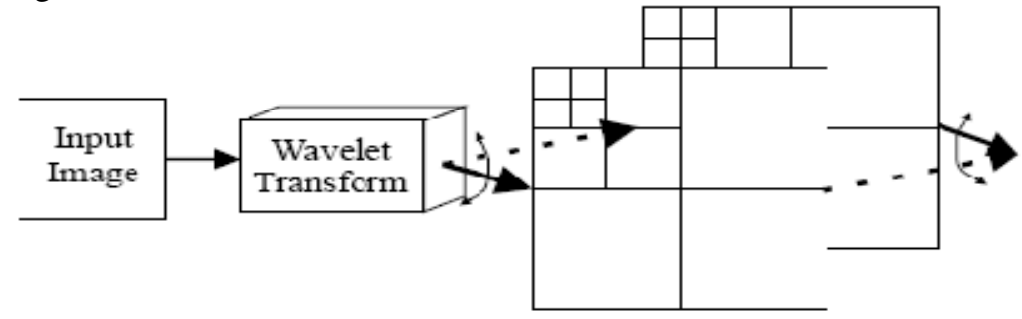

Figure 1. Wavelet transform diagram [6]

The Discrete Wavelet Transform (DWT) techniques have been resolved by Creusere (1997) to decompose discrete time signals. The analysis scheme is named asub-band coding. Burt (1983) determined a technique like sub-band coding and called it pyramidal coding which is also famed as multi-resolution analysis. Later Vetterli et al (1990) made some improvements by removing the existing redundancy in the pyramidal coding scheme. The basic idea of the DWT for a twodimensional image is termed as follows. An image is first decomposed into four parts based on frequency sub bands, by critically sub sampling horizontal and vertical channels using sub band filters and named as Low-Low (LL), Low-High (LH), High-Low (HL), and High- High (HH) sub bands. To obtain the next coarser we will further decompose LL subband. This process is repeated several times, figure 2 shows it.

\begin{tabular}{|l|l|l|l|}
\hline LL & HL & & \\
\cline { 1 - 2 } LH & HH & HL2 & \\
\cline { 1 - 2 } LH2 & HH2 & HL1 \\
\hline \multicolumn{2}{|l|}{ LH1 } & & HH1 \\
\hline
\end{tabular}

Figure 2. Three scale wavelet decomposition [7] 
Signal \& Image Processing : An International Journal (SIPIJ) Vol.5, No.6, December 2014

The image of low frequency sub-band contains major information. High frequency sub-band values approximate zero, the more high frequency the more obvious this situation. In the image, the low frequency part acts the image information. So researchers take full advantage of the characteristic after wavelet transform and employ proper method to process the image coefficients for achieving further compression.

\section{EZW ALgORITHM}

The embedded zero tree wavelet algorithm (EZW) [7] is an effective image compression algorithm. a fully embedded bit stream for image coding by this new technique. EZW algorithm is an evident advantage. The user can select a bit rate and encode[8] the image to exactly the wanted bit rate.

Features of EZW algorithm:

(1) Compact binary maps are provided by zero tree coding of significant wavelet coefficients.

(2) Successive approximation quantization of wavelet coefficients.

(3) Huffman coding.

\subsection{Embedded Coding}

The embedded zerotree wavelet algorithm (EZW) is a simple and efficient image compression algorithm. [9] The bits in the bit stream are generated in order of importance in this coding algorithm. [10] The schematic diagram of an makes the image transform, can be shown in Figure 3. The image coder has three main blocks: transform, quantizer, and entropy coder .

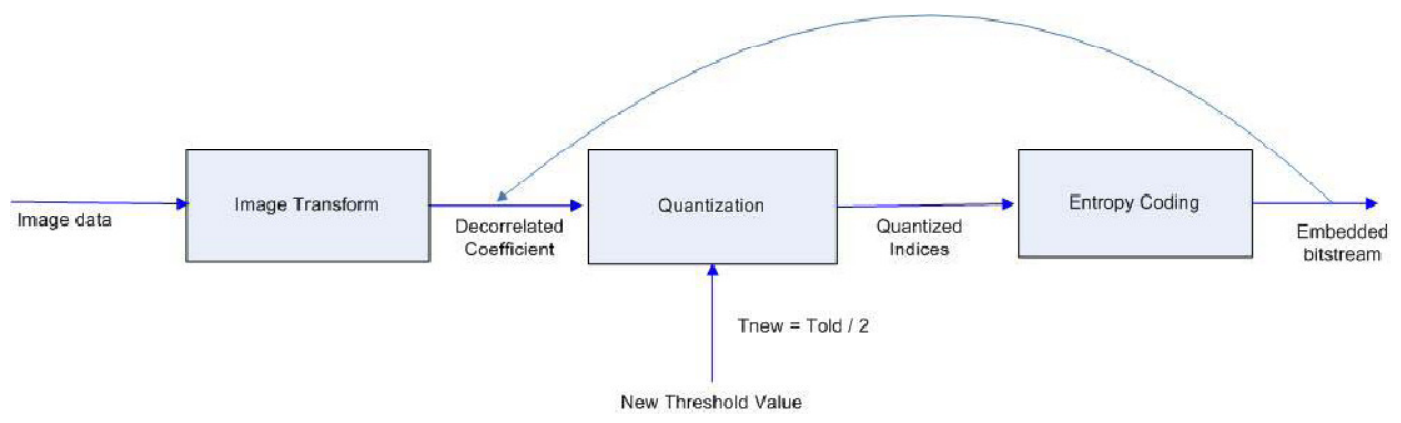

Figure 3. Embedded coding scheme [10]

In the case of the DWT (image transform), image is decomposed and producing the wavelet coefficients at different scales. We use next block in Figure is a quantizer. It quantizes the transformed matrix into a sequence of integers. After that using an entropy coding scheme.

In addition, The EZW encoder is based on two important annotations:

(1) The progressive encoding is a very normal option for compressing wavelet transformed images, since the higher subbands only add detail.

(2) Large wavelet coefficients are more substantial than smaller wavelet coefficients. Shapiro is a person who introduced Embedded zero-tree coding of wavelet coefficients (EZW). In the Ezw algorithm if a wavelet coefficient at a coarse scale is insignificant with ratingto a threshold $\mathrm{T}$, then all wavelet coefficients of the same orientation in the same spatial location at finer scale will be insignificant with respect To T. The trees induce a parent-child relationship among the coefficients of subbands having the same spatial 
Signal \& Image Processing : An International Journal (SIPIJ) Vol.5, No.6, December 2014

orientation. These parent-child dependencies are at all events credited for the excellent performance of zero-tree coders. Figure 4 shows The coarse scale coefficient is called the parent and all coefficients corresponding to the same spatial location at the next finer scale of similar orientation are called children .
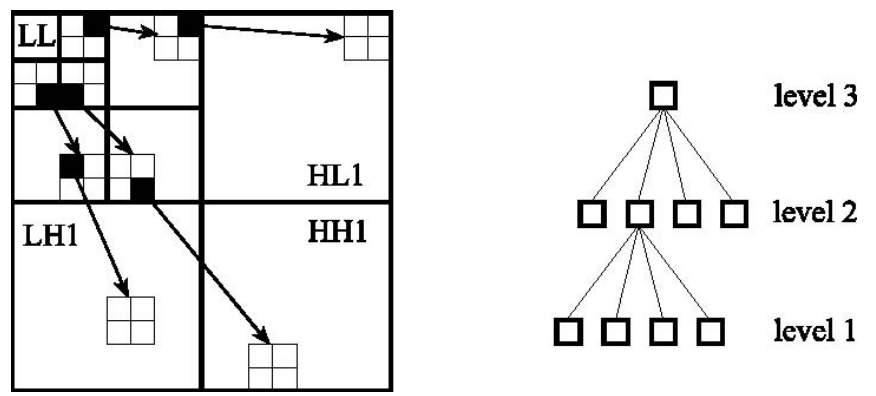

Figure 4. Parent-child dependencies [10]

We summarize the EZW coding algorithm as follows: The EZW output stream will have to begin with some information to synchronize the decoder. The minimum information required by the decoder needs the number of wavelet transform levels used and the initial threshold, if we suppose that always the same wavelet transform will be used. After imperfect reconstruction the decoder can then replace the incomplete mean by the original mean. This can raise the PSNR significantly. The EZW coding algorithm includes the first step to determine the initial threshold. If we choose bitplane coding then our initial threshold t0 will be

$$
t_{0}=2^{\left\lfloor\log _{2}(\operatorname{MAX}(|y(x, y)|))\right\rfloor}
$$

$\operatorname{MAX}(\gamma(\mathrm{x}, \mathrm{y}))$ is the maximum coefficient value in the image with this threshold. we enter the main coding loop.

$$
\begin{aligned}
& \text { threshold = initial_threshold; } \\
& \text { do }\{ \\
& \text { dominant_pass(image); } \\
& \text { subordinate_pass(image); } \\
& \text { threshold = threshold/2; } \\
& \text { \} while (threshold > minimum_threshold); }
\end{aligned}
$$

We see that the image is coded by using two passes. the dominant pass is the first pass, a symbol is outputted for every coefficient after scanning the image. If the coefficient is larger than the threshold a $\mathrm{P}$ (positive) is coded, if the coefficient is smaller than minus the threshold an $\mathrm{N}$ (negative) is coded. If the coefficient is the root of a zerotree then a T (zerotree) is coded and finally, if the coefficient is smaller than the threshold but it is not the root of a zerotree, then a $\mathrm{Z}$ (isolated zero) is coded. This happens when there is a coefficient larger than the threshold in the tree. The effect of using the $\mathrm{N}$ and $\mathrm{P}$ codes is that when a coefficient is found to be larger than the threshold (in absolute value or magnitude) its two most significant bits are outputted (if we forget about sign extension). Note that in order to determine if a coefficient is the root of a zerotree or an 
Signal \& Image Processing : An International Journal (SIPIJ) Vol.5, No.6, December 2014

isolated zero, the subordinate pass is the second pass and is called the refinement pass. In this list is ordered so that the largest coefficients are again transmitted first. The main loop stops when the threshold reaches a minimum value. For integer coefficients is minimum value equals zero and the divide by two and after that can be replaced by a shift right operation. If we append another ending condition based on the number of outputted bits by the huffman coder then we can apply any target bit rate exactly without doing too much work.

We abstracted the above with the following code fragments, starting with the dominant pass. In the dominant_pass as shown in figure 5:

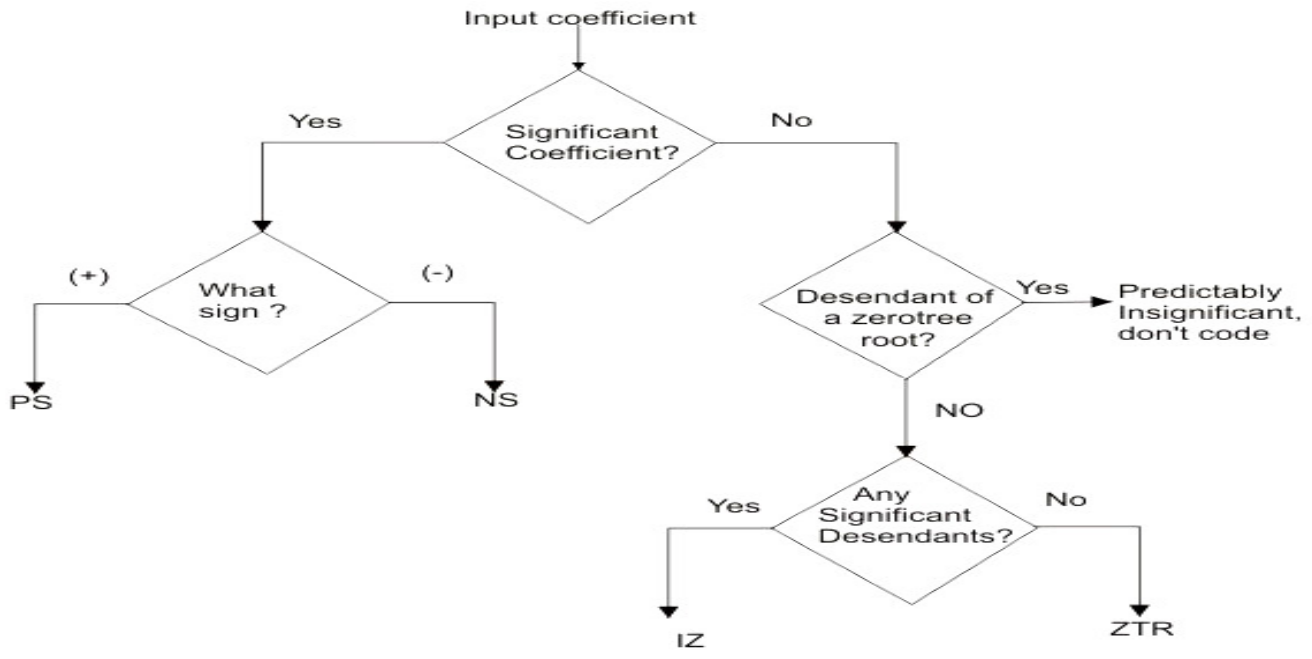

Figure 5. Flowchart for dominant pass [10]

In the dominant_pass : all the coefficients are larger than the current threshold are extracted and placed without their sign on the subordinate list after that we will make their positions in the image are filled with zeroes. This will make them from being coded still.

In the subordinate_pass : All the coefficients in the subordinate list are refined. this gives boost to some juggling with uncertainty intervals and it products next most significant bit of all the coefficients in the subordinate list

\section{SIMULATED RESULTS}

We stratified the embedded zero-tree wavelet algorithm (EZW) on the lena image. The input image file is used in BMP format. The image size was considered to be $512 * 512$ pixels. , the image was compressed and reconstructed by using a threshold value of 8 for EZW algorithm and using Huffman coding. The compression ratio and the calculated PSNR value determined for the image Lena in table 1.

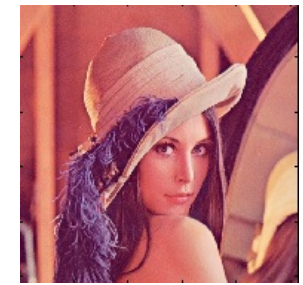

Figure 6. Original image

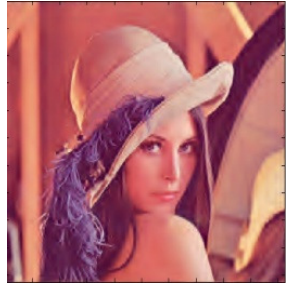

Figure 7. EZW reconstructed image 
Signal \& Image Processing : An International Journal (SIPIJ) Vol.5, No.6, December 2014

Table 1. Performance evaluation of dwt-ezw algorithm

\begin{tabular}{|c|c|c|}
\hline Technique & CR & PSNR(dB) \\
\hline Ezw & 1.6647 & 30.46 \\
\hline
\end{tabular}

\section{CONCLUSION \& FUTURE WORK}

This paper is about Embedded Zerotree Wavelet (EZW) algorithm which is very much indeed effective and important compression algorithm. This algorithm is invent by Shapiro in (1993) and it is an image compression algorithm that the bits in the bit stream are produced in order of importance. The algorithm is dependent on the selection of significant coefficients with respect to given thresholds. In this paper, we tried to use the embedded zero tree wavelet compression on color Lena image and the PSNR and compression ratio values fixed for the Image.

In the future work we will make a comparison between EZW (Embedded Zero tree wavelet) and SPIHT (Set Partitioning in Hierarchical Trees) coding techniques in color image compression.

\section{REFERENCES}

[1] R.C. Gonzalez, R. E. Woods, and S..L. Eddins.' 'Digital Image Processing Using MATLAB”.. ISBN10:0130085197. ISBN-13: 978-0130085191. Prentice Hall, 1st edition ,September 5, 2003.

[2] P. Singh, Nivedita and S.Sharma, "A Comparative Study: Block Truncation Coding, Wavelet, Embedded Zerotree and Fractal Image Compression on ColorImage", International Journal of Electronics and Communication Engineering \& Technology(IJECET), Volume 3, Issue 2, ISSN Print: 0976- 6464, ISSN Online:0976 -6472, pp. 10 - 21,2012.

[3] R. A. DeVore, B. Jawerth, and B. J. Lucier, "Image Compression Through Wavelet Transform Coding," IEEE Trans. On Information Theory, Vol. 38, No. 2,pp. 719 - 746, 1992.

[4] H. J. Barnard, Image and Video Coding Using Wavelet Transforms, Ph.D. Dissertation, Technische Universiteit Delft, May 1994.

[5] N. Ahmed, T. Natarajan, and K. R. Rao, Discrete Cosine Transform, IEEE. Trans. Computer, Vol C-23, pp. 90-93 Jan 1974.

[6] S. Pemmaraju and S. Mitra, "Efficient image coding using multiresolution wavelet transform and vector quantization" IEEE Southwest Symposium on Image Analysis and Interpretation, IEEE press, Santa Fe, New Mexico, pp:243-248,April, 1996.

[7] J.M.Shapiro, "Embedded Image Coding Using Zerotrees of Wavelet Coefficients", IEEE Trans. on Signal Processing, Vol. 41, pp 3445-3462, 1993.

[8] R. Ponalagusamy and C .Saravanan," Analysis of MedicalImage Compression using Statistical Coding Methods, Advances inComputer Science and Engineering: Reports and Monographs,Imperial College Press, UK, Vol.2., pp 372-376, 2007.

[9] A. S. Lewis and G. Knowles, "Image Compression Using the 2-D Wavelet Transform", IEEE Trans. On Image Processing, Vol. 1, No. 2, pp. 244-250, April, 1992.

[10] V.S.Shingate, T.R. Sotakke and S.N.Talbar, "Still Image Compression using Embedded Zerotree Wavelet Encoding”, Inrenational Journal of Computer Science \& Communication, Vol.1,No.1,,pp.2124 January-June 2010. 